\title{
On the Estimation of Interleaved Pulse Train Phases
}

\author{
Tanya L. Conroy and John B. Moore, Fellow, IEEE
}

\begin{abstract}
Some signals are transmitted as periodic pulse trains where information is in the timing of the arrival of the pulses. A number of pulse trains arriving over the same time interval are said to be interleaved. We propose an on-line method for estimating pulse train phases and fine-tuning pulse repetition frequency (PRF) estimates of a known number of interleaved pulse trains. The computational effort is of order $N$, where $N$ is the number of pulses received. In particular, we employ an extended Kalman filter, where discontinuities in the signal model are appropriately smoothed.
\end{abstract}

Index Terms—Phase estimation, pulse train deinterleaving.

\section{INTRODUCTION}

$\mathbf{S}$ OME signals occur as periodic trains of pulses. For example, signals used in radar systems, communication systems, and possibly neural systems appear in this form. Often, a number of pulse trains are received over a single channel during the same time interval, resulting in an interleaved pulse train. It is important to be able to separate the pulses in the interleaved train in terms of their source in order to extract desired information. This process is termed pulse train deinterleaving. One application for pulse train deinterleaving is in radar detection [1].

Previously proposed pulse train deinterleaving methods include sequential search [2] and histogramming [2], [3], which work well in low-noise environments. Another approach is first to formulate the problem as a stochastic discrete-time dynamic linear model and then deinterleave the signal using either forward dynamic programming with fixed look-ahead or a probabilistic teacher [4]. All these methods are computationally expensive. If the pulse train to be deinterleaved contains $N$ pulses, then these methods require a computational effort of order $N^{2}$ or higher. It could be argued that if $N$ is, say, 100 to 300 pulses, then the $O\left(N^{2}\right)$ is not unattractive, but if $N$ is say, 10000 , then $O\left(N^{2}\right)$ is not tractable for real time implementation.

An extended Kalman filter [5] approach to deinterleaving using a modified version of the signal model in [4] is presented in [6]. Here, the computational effort required is of order $N$. Another method for deinterleaving using the Kalman filter derived from techniques for multiple target tracking is presented in [7], where a maximum of three trains are present in the interleaved train.

The deinterleaving task relies on the assumption that the different trains will have different pulse repetition frequencies

\footnotetext{
Manuscript received May 5, 1999; revised August 8, 2000. The associate editor coordinating the review of this paper and approving it for publication was Dr. Vikram Krishnamurthy.

The authors are with the Department of Systems Engineering, Research School of Information Sciences and Engineering, Australian National University, Canberra, Australia (e-mail: Tanya.Conroy@syseng.anu.edu.au).

Publisher Item Identifier S 1053-587X(00)10162-X.
}

(PRFs) and phases; therefore, the estimation of these characteristics is perhaps a useful starting point. One computationally efficient method for this uses fast Fourier transform techniques to determine the number of trains present and their PRFs but does not deinterleave the trains [8]. The computational effort required is of order $N \log N$. A state-space method for parameter estimation is given in [9] to estimate the pulse repetition interval and the variance of the jitter noise of a single pulse train.

None of the above methods seek to directly estimate the phases of the interleaved pulse trains. In this paper, a new signal model for use with an extended Kalman filter is proposed. As with [8], we do not deinterleave the pulse trains but estimate their characteristics. Here, we directly estimate the phases and fine tune the PRF estimates obtained using the method in [8] of the interleaved pulse trains with computational effort of order $N$. It is assumed that the pulse trains are periodic and that the number of sources is finite and known (also from spectral analysis [8]).

\section{SigNAL MODEL}

Consider $M$ periodic pulse train sources. Let $f^{(i)}$ and $\theta^{(i)}$ denote the PRF and phase of the $i$ th source. Note that the pulse train phases take values in the interval $[0,2 \pi)$. Pulses then occur at times $\left(2 \pi n+\theta^{(i)}\right) /\left(2 \pi f^{(i)}\right)$ for $i \in[1, M]$ and $n=0,1,2, \cdots$ The received interleaved signal consists of the superposition of the $M$ pulse trains produced by these sources. Let $t_{1}, t_{2}, \cdots, t_{N}$ denote the times of arrival of $N$ consecutive pulses. The deinterleaving problem is as follows.

Deinterleaving Task: Given pulse arrival times $t_{1}, \cdots, t_{N}$ and the number of sources present $M$, determine which source produced each pulse.

A first step toward solving this is phase/PRF estimation.

Phase/PRF Estimation Task: Given pulse arrival times $t_{1}, \cdots, t_{N}$ and the number of sources present $M$, estimate the frequencies $f^{(i)}$ and phases $\theta^{(i)}$ of each pulse train $i=1,2, \cdots, M$.

Since our computer implementation of the estimation algorithms are in discrete time, it makes sense to work with discrete-time models that are fast sampled approximate versions of the precise continuous-time models. The signal can be described by a discrete-time model

$$
\begin{aligned}
x_{k+1} & =x_{k}+v_{k}, x_{0} \\
y_{k} & =h_{k}+w_{k} .
\end{aligned}
$$

Here, $k$ is the discrete-time index, and the received signal $y_{k}$ is the number of pulses detected before the discrete time step $k$. In 
addition, $x_{k}$ is the state variable at $k$ with elements of the PRFs $f^{(i)}$ and phases $\theta^{(i)}$ as follows:

$$
x_{k}^{\prime}=\left[f_{k}^{(1)}, \cdots, f_{k}^{(M)}, \theta_{k}^{(1)}, \cdots, \theta_{k}^{(M)}\right]
$$

where there are $M$ pulse train sources, $f_{k}^{(i)}$ is the PRF, and $\theta_{k}^{(i)}$ is the phase of train $i$, as shown in Fig. 1 . The terms $v_{k}$ and $w_{k}$ represent noise on the states and received signal.

The received signal $h_{k}$ is the number of pulses that have arrived in the interleaved train at time $k$. A typical example of $h_{k}$ for $M=4$ is shown in Fig. 2, where jumps occur when a pulse is received. Note that the jumps are not instantaneous due to the discrete nature of the model. A pulse is only known to arrive within a discrete time period rather than at an exact time. Thus, $h_{k}$ can be expressed in terms of the state as follows:

$$
\begin{aligned}
h_{k}^{(i)} & =f^{(i)} k-\theta^{(i)} / 2 \pi-r_{k}^{(i)} \\
h_{k} & =\sum_{i=1}^{M} h_{k}^{(i)}
\end{aligned}
$$

where $r_{k}^{(i)}$ is a minimum value remainder term that ensures that $h_{k}^{(i)}$ is an integer. The remainder term can also be related to the state since for each pulse train at each time instant, $r_{k}^{(i)}$ is equal to the fractional part of $f^{(i)} k-\theta^{(i)} / 2 \pi$.

The terms $v_{k}, w_{k}$ in (1) represent noise on the states and received signal, respectively. Thus, $v_{k}$ represents drifting of phase and frequency or, equivalently, cumulative jitter on the states, and $w_{k}$ represents noncumulative jitter, which translates to a variation in the number of pulses observed at time $k$ and could also be used to represent the number of false detections of pulses or missing pulses. For simplicity, we assume that $v_{k}$ and $w_{k}$ are independent, zero mean, and Gaussian with covariances $Q_{k}$ and $R_{k}$, respectively. Since the received signal $y_{k}$ is an integer, $w_{k}$ must, in reality, be integer-valued noise, but as Fig. 3 shows, for Gaussian noncumulative jitter added to the pulse arrival times, the noise distribution on the received signal is also Gaussian.

It can be seen from (3) that $h_{k}$ depends nonlinearly on the state; therefore, the state space model (1) is nonlinear. The nonlinearities in $h_{k}$ are discontinuous; therefore, the signal model cannot be used in its present form to derive an extended Kalman filter $(\mathrm{EKF})$.

Remark: In [6], a discrete-event state-space model is formulated with the "discrete-time" variable $k$ being the integer number of pulses that have arrived. This method is of order $N$. Here, $k$ depends on the rate at which the interleaved train is sampled. Since the sample rate only linearly increases the order of the deinterleaver, this method is also of order $N$ for any reasonable sample rate. In the simulations that follow, we use a sample rate of $100 \mathrm{kHz}$, which is about 10 times higher than the highest PRF present. It is important only that this rate be fast enough to accurately capture the interleaved train and slow enough not to cause instability in the EKF. This instability occurs when the input to the EKF varies infrequently in comparison to the sampling rate.

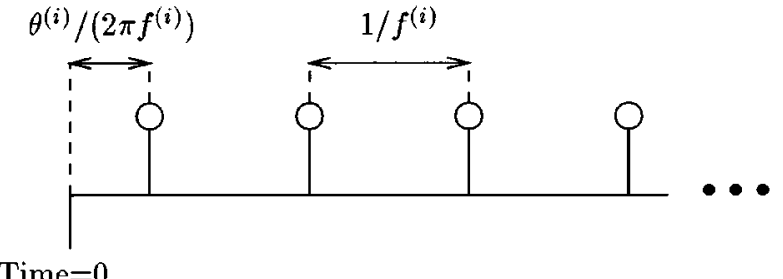

Fig. 1. Single pulse train and its characteristics.

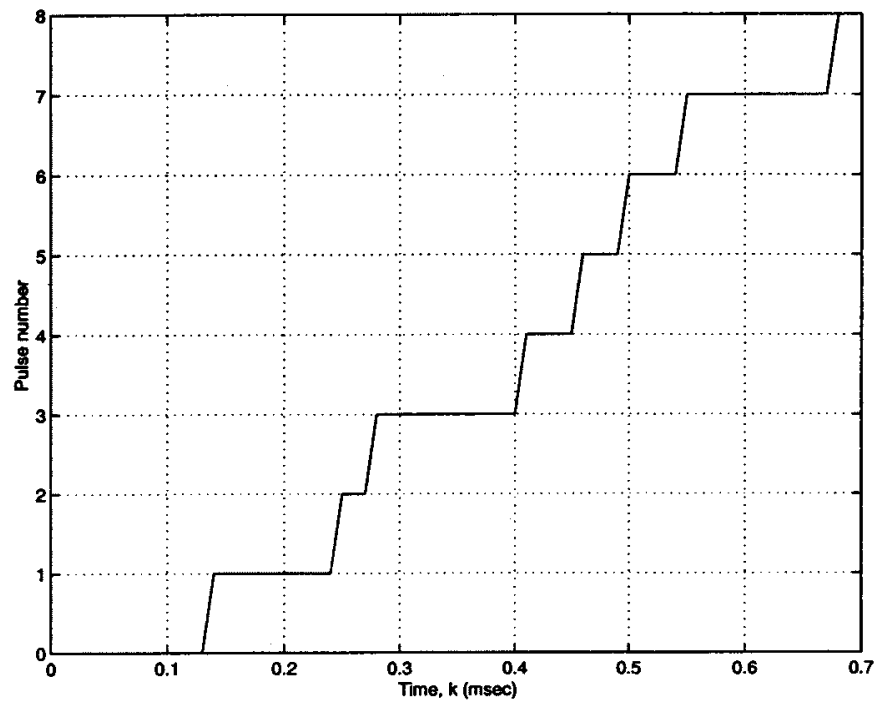

Fig. 2. Received signal $h_{k}^{(i)}$.

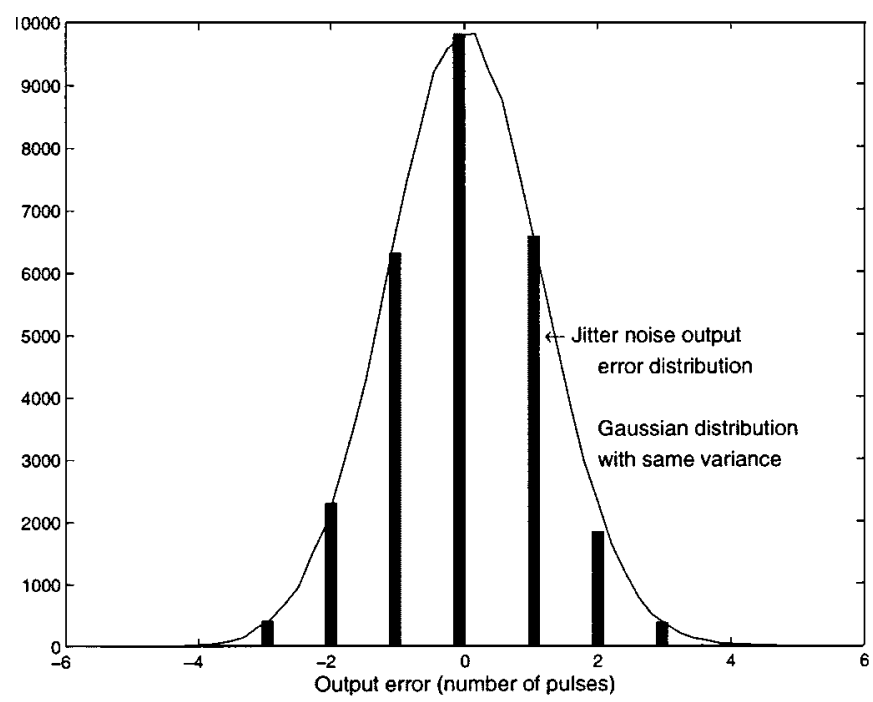

Fig. 3. Distribution of noise on the received signal $y_{k}$ in the presence of noncumulative Gaussian jitter noise $\left(\sigma^{2}=10 n s^{2}\right)$ on the pulse times of arrival. The output noise variance in this case is 1.35 pulses.

\section{A. Smoothing $h_{k}$}

A key proposal of this paper is to exploit the extended Kalman filter in some way for recursively estimating the states (phases and PRFs of the pulse trains) from the data $y_{k}$. This is a first step toward deinterleaving. In order to use an EKF for deinterleaving, our approach is to smooth the nonlinearities inherent in the signal $h_{k}$ so that these can be linearized. This can be done, 


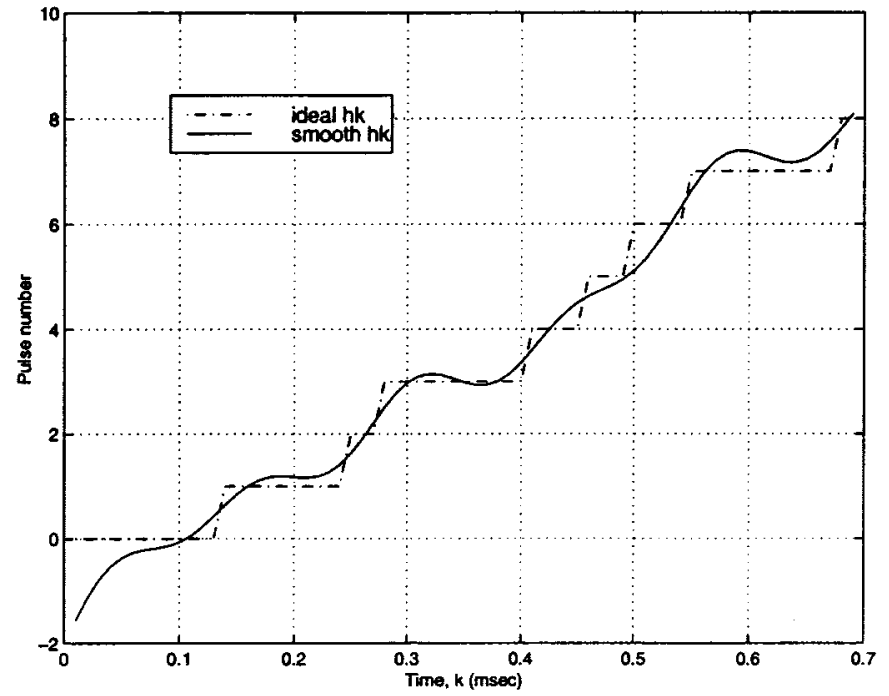

Fig. 4. Smoothed signal $h_{k}^{s}$.

for example, by truncating a Fourier series expansion of $h_{k}$ to $A$ terms. The resultant expression for $h_{k}$ is

$$
\begin{aligned}
h_{k}^{s(i)}= & \left(f_{k}^{(i)} k-\theta_{k}^{(i)} / 2 \pi-1 / 2\right) \\
& +\sum_{a=1}^{A} \sin \left(2 \pi a f_{k}^{(i)} k-a \theta_{k}^{(i)}\right) / a \pi \\
h_{k}^{s}= & \sum_{i=1}^{M} h_{k}^{s(i)} .
\end{aligned}
$$

In practice, the first term of the Fourier series $[A=1$ in (4a)] appears sufficient to approximate the original discontinuous signal for filtering purposes. As $A$ increases, the smooth signal better approximates the original signal, becoming less smooth with each term added. This is undesirable in this case where a smooth approximate signal is needed for use in the EKF. Fig. 4 shows the smoothed version of the signal in Fig. 2.

The smoothed signal model is now

$$
\begin{aligned}
x_{k+1} & =x_{k}+v_{k} \quad, x_{0} \\
y_{k} & =h_{k}^{s}\left(x_{k}\right)+w_{k}
\end{aligned}
$$

where $h_{k}^{s}\left(x_{k}\right)$ is given in (4).

\section{EXTENDED KALMAN FiLTeR}

In order to construct an EKF from the signal model given in (5), a linearization of $h_{k}^{s}\left(x_{k}\right)$ (4) is needed. This is

$$
H_{k}^{\prime s}=\left[\cdots, \partial h_{k}^{s}\left(x_{k}\right) / \partial f_{k}^{(i)}, \cdots, \partial h_{k}^{s}\left(x_{k}\right) / \partial \theta_{k}^{(i)}, \cdots\right]
$$

where

$$
\begin{aligned}
& \frac{\partial h_{k}^{s}\left(x_{k}\right)}{\partial f_{k}^{(i)}}=k+2 k \cos \left(2 \pi f_{k}^{(i)} k-\theta_{k}^{(i)}\right) \\
& \frac{\partial h_{k}^{s}\left(x_{k}\right)}{\partial \theta_{k}^{(i)}}=-\frac{1}{2 \pi}-\frac{1}{\pi} \cos \left(2 \pi f_{k}^{(i)} k-\theta_{k}^{(i)}\right) .
\end{aligned}
$$

The EKF equations are simplified by the constant state equation in the signal model. This yields constant time-update equations, and therefore, the filter equations are

$$
\begin{aligned}
\hat{x}_{k+1 / k} & =\hat{x}_{k / k-1}+K_{k}\left[y_{k}-h_{k}\left(\hat{x}_{k / k-1}\right)\right] \\
K_{k} & =P_{k / k-1} H_{k}^{s}\left(H_{k}^{\prime s} P_{k / k-1} H_{k}^{s}+R_{k}\right)^{-1} \\
P_{k+1 / k} & =P_{k / k-1}-K_{k} H_{k}^{\prime s} P_{k / k-1}+Q_{k}
\end{aligned}
$$

where

$\hat{x}_{k+1 / k} \quad$ filtered estimate of $x_{k+1}$;

$K_{k} \quad$ Kalman gain;

$P_{k / k-1}$ error covariance matrix at $k$ given measurements to $k-1$.

Notice that the nonsmooth estimate of the input signal $h_{k}\left(\hat{x}_{k / k-1}\right)$ is used in the state update equation. The initialization here is given by $\hat{x}_{0 /-1}=\hat{x}_{0}$, where $\hat{x}_{0}=\left[\begin{array}{ll}\hat{f}^{\prime} & \hat{\theta}_{0}^{\prime}\end{array}\right]^{\prime}$, and $P_{0 /-1}=P_{0}$.

Remark: The EKF is near optimal under the following conditions:

- good initial estimates of the parameters;

- low noise;

- good approximations of the smoothed nonlinearities.

When operating outside these conditions, there is no theory that states how well it will operate. See [5] for further information on the EKF.

\section{RESULTS}

Ten sets of randomly generated PRFs and phases were used to construct interleaved pulse trains with various numbers of component trains to test the effectiveness of the EKF parameter estimation method. There was no noise on the interleaved trains. It was found that in eight out of the ten cases, the EKF parameter estimator could fine tune the PRF estimates and estimate the phases of up to seven interleaved pulse trains after processing an interleaved train with 2000 pulses. The mean error in the PRF and phase estimates over the eight cases was $13.8 \mathrm{e}-3 \%$ and $2.0 \%$, respectively. The other two cases are dealt with in the section on estimation failure below.

Fig. 5 shows typical results for the PRF fine tuning and phase estimation from the EKF with no noise on the interleaved train. The interleaved train input consists of 2000 pulses $(N=2000)$ with five component pulse trains $(M=5)$. The estimation results are as shown in Tables I and II.

These results are obtained with an initial $10 \%$ uncertainty in the PRF's and unknown phases on the interval $[0,2 \pi)$. The initialization matrices used reflect this. Here, $\hat{x}_{0}$ is given by $\hat{f}_{0}^{(i)}=0.9 f^{(i)}$ (by spectral studies [8]) and $\theta_{0}^{(i)}$ randomly selected from a normal distribution over $[0,2 \pi)$. The initialization for the error covariance matrix $P_{0}$ is therefore given as follows:

$$
P_{0}=\left[\begin{array}{cc}
\operatorname{diag}\left(\left(\hat{f}^{(i)}\right)^{2} / 100\right) & 0_{M \times M} \\
0_{M \times M} & \operatorname{diag}\left(\pi^{2} / 3\right)
\end{array}\right] .
$$

The noise covariance $R_{k}$ was set to 10 to add some robustness to modeling uncertainties. $Q_{k}$ was set to zero because there is no cumulative jitter present in the system. 

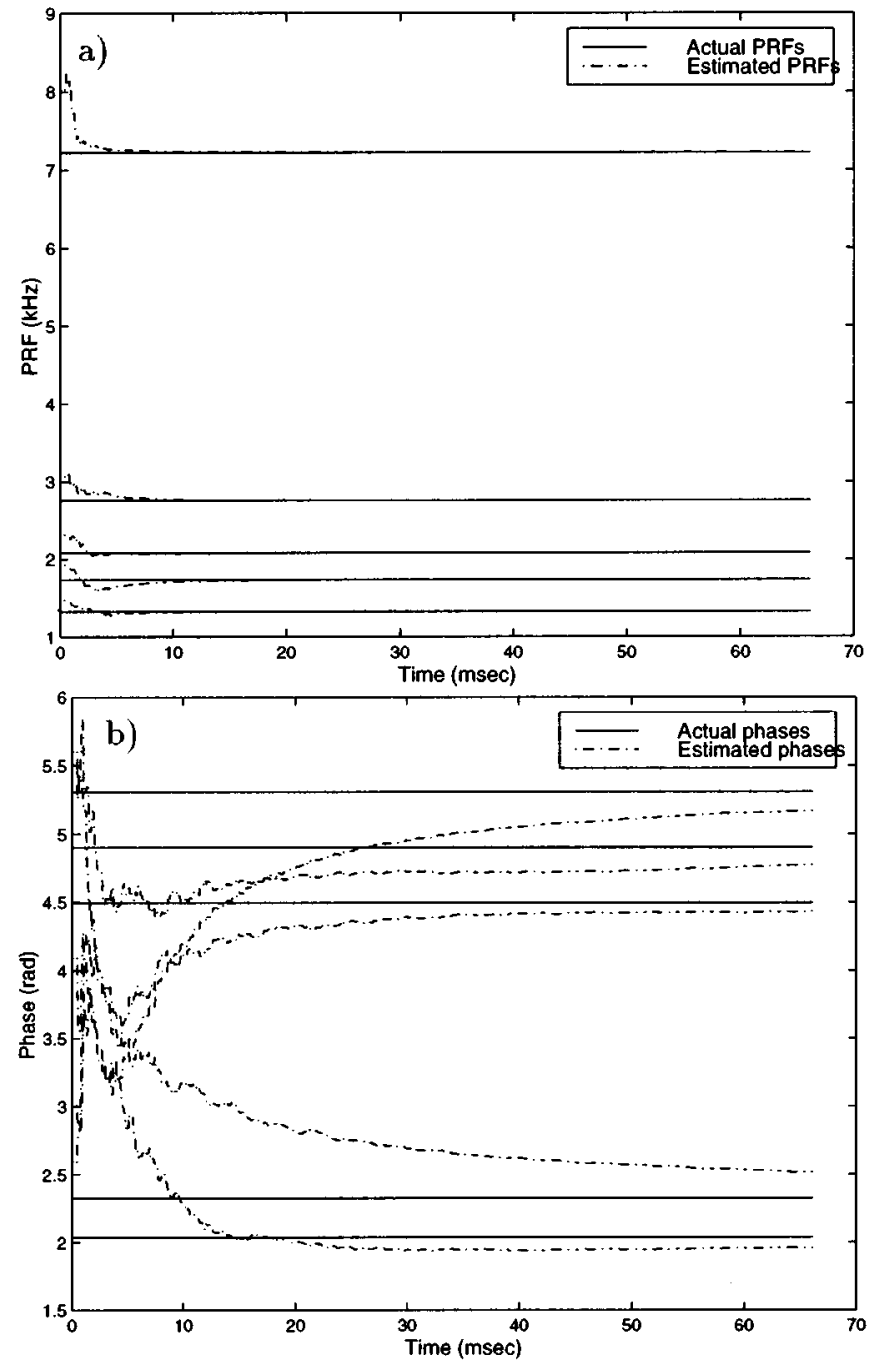

Fig. 5. Parameter estimation over 1000 pulses. (a) Actual and estimated PRFs. (b) Actual and estimated phases.

TABLE I

COMPARISON OF PRFS

\begin{tabular}{cccc}
\hline Train No. & $\begin{array}{c}\text { Actual PRF } \\
(\mathrm{kHz})\end{array}$ & $\begin{array}{c}\text { Est. PRF } \\
(\mathrm{kHz})\end{array}$ & $\begin{array}{c}\text { Error } \\
(\%)\end{array}$ \\
\hline 1 & 1.3238 & 1.3237 & $4.8 \mathrm{e}-3$ \\
2 & 1.7364 & 1.7363 & $6.3 \mathrm{e}-3$ \\
3 & 2.0830 & 2.0828 & $5.8 \mathrm{e}-3$ \\
4 & 2.7595 & 2.7595 & $1.5 \mathrm{e}-3$ \\
5 & 7.2244 & 7.2245 & $2.5 \mathrm{e}-3$ \\
\hline
\end{tabular}

TABLE II

COMPARISON OF PHASES

\begin{tabular}{cccc}
\hline Train No. & $\begin{array}{c}\text { Actual Phase } \\
\text { (rad) }\end{array}$ & $\begin{array}{c}\text { Est. Phase } \\
\text { (rad) }\end{array}$ & $\begin{array}{c}\text { Error } \\
\text { (\%) }\end{array}$ \\
\hline 1 & 4.50 & 4.46 & 0.6 \\
2 & 5.31 & 5.25 & 1.0 \\
3 & 4.90 & 4.82 & 1.3 \\
4 & 2.04 & 1.98 & 1.0 \\
5 & 2.33 & 2.41 & 1.4 \\
\hline
\end{tabular}

As can be seen from Table I, the fine tuning of the PRF is very effective. Table II indicates that there can be some bias in estimating the phase (due to inaccuracies in the model and the effect of the positive definite $R_{k}$ ) but that this bias is small. Processing more pulses slightly improves the phase estimation, but beyond around 2000 pulses, this improvement is minimal.

Of course, one would not be surprised if a method involving of order $N^{2}$ calculations (or higher) achieved similar, or better results, but the main message from this work is that very useful results are achieved using a method requiring only of order $N$ calculations.

\section{A. Sensitivity to Initial Conditions}

As with [6], or any method that uses an EKF for estimation or deinterleaving, this parameter estimation method is sensitive to initial conditions chosen. Here, an initial estimate for PRF is known (perhaps using the method of [8]) and assumed to be within $10 \%$ of the true value. The difficulty lies in choosing an initial value for the phases.

Since the pulse train phases are assumed to lie on the interval $[0,2 \pi)$, the initial estimates for the phases are randomly chosen from a uniform distribution over this interval. It makes sense then to use a bank of filters, each with a different initialization for phase, with the filter leading to the least average prediction error squared being chosen. Since this method is of order $N$, it will retain its computational advantage over most previous deinterleaving methods if the number of filters in the bank is much less than $N$.

\section{B. Jitter Noise}

Noise is present in all real-world signals, so it is important to test this pulse train parameter estimation method in the presence of noise. Here, we examine only the case where white Gaussian noise with known variance is present. It could be possible to deal with noise of unknown variance or bias using an adaptive Kalman filter, but this is beyond the scope of this paper.

Time-of-arrival jitter noise with variance $\sigma^{2}=$ $1 \mathrm{~ns}^{2}, 2 \mathrm{~ns}^{2}, 5 \mathrm{~ns}^{2}$ and $10 \mathrm{~ns}^{2}$ was added to the interleaved pulse train. This noise is noncumulative jitter. $R_{k}$ is set to 10 when $\sigma^{2}=1 \mathrm{~ns}^{2}$ or $2 \mathrm{~ns}^{2}$ and 20 when $\sigma^{2}=5 \mathrm{~ns}^{2}$ or $10 \mathrm{~ns}^{2}$. As with the noise-free case, $Q_{k}$ is zero as there is no noncumulative jitter present. To determine how significant these noise levels are when applied to the pulse trains in the simulation, the noise standard deviation is compared with the period of the highest frequency train. Here, that train has a pulse repetition interval (PRI) of $T_{\mathrm{MIN}}=0.138 \mathrm{~ms}$ so that when $\sigma^{2}=1 \mathrm{~ns}^{2}$

$$
\frac{\sigma}{T_{\mathrm{MIN}}}=0.2285
$$

or the standard deviation of the jitter is $23 \%$ of the smallest period present. When $\sigma^{2}=2 \mathrm{~ns}^{2}, 5 \mathrm{~ns}^{2}$ and $10 \mathrm{~ns}^{2}$, the standard deviation of the jitter is $32 \%, 51 \%$, and $72 \%$ of the smallest period, respectively.

Fig. 6 shows how the performance of the system degrades as jitter noise is increased. Here, we assume that the parameters of a set of pulse trains have been successfully estimated if the average phase error is within $10 \%$. Table III shows the average PRF and phase errors for the maximum number of trains estimated at each noise level. 


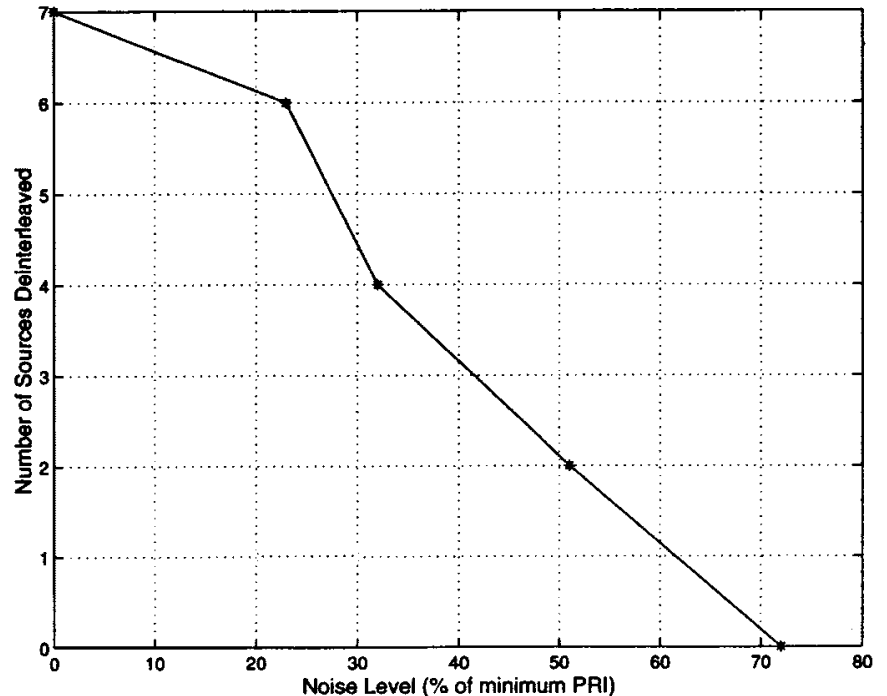

Fig. 6. Degradation of algorithm with increasing noise.

TABLE III

Average PRF and Phase ERrors for a Typical Set of Pulse Trains UNDER VARIOUS NOISE CONDITIONS

\begin{tabular}{cccc}
\hline $\begin{array}{c}\text { Noise } \\
\text { \% lowest PRI }\end{array}$ & $\begin{array}{c}\text { No. of } \\
\text { Trains }\end{array}$ & $\begin{array}{c}\text { Average PRF } \\
\text { Error (\%) }\end{array}$ & $\begin{array}{c}\text { Average Phase } \\
\text { Error (\%) }\end{array}$ \\
\hline $0 \%$ & 7 & $12.7 \mathrm{e}-3$ & 2.1 \\
$23 \%$ & 6 & $20.1 \mathrm{e}-3$ & 4.0 \\
$32 \%$ & 4 & $37.3 \mathrm{e}-3$ & 8.2 \\
$51 \%$ & 2 & $7.7 \mathrm{e}-3$ & 7.6 \\
$72 \%$ & 0 & - & - \\
\hline
\end{tabular}

Fig. 7 shows the effect of jitter noise with variance $1 \mathrm{~ns}^{2}$ on the same five train system as shown in Fig. 5, where no noise is present.

\section{Estimation Failure}

Any method for time-of-arrival pulse train deinterleaving or parameter estimation will fail if the pulse environment is too dense, i.e., there are too many pulse trains being received during the same time period over the same channel. This causes this method to fail for more than seven pulse trains in the received signal. Similarly, no method works well when there is too much noise on the received signal.

There are some cases where, even without noise on the system, and with the presence of a bank of EKF estimators to eliminate difficulties due to the sensitivity of the EKF to initial conditions, the phase and PRFs of a set of trains are not correctly estimated. This occurs primarily when two trains in the interleaved train have PRFs and phases that are very similar. In this case, even if the other trains are correctly estimated, these two trains are not due to their similarity. See Tables IV and $\mathrm{V}$ for an example where this is the case. Here, pulse trains 1 and 2 are very similar, and estimation fails. This case is likely to prove to be a problem for any estimation or deinterleaving method as the two trains interfere with each other at every pulse.
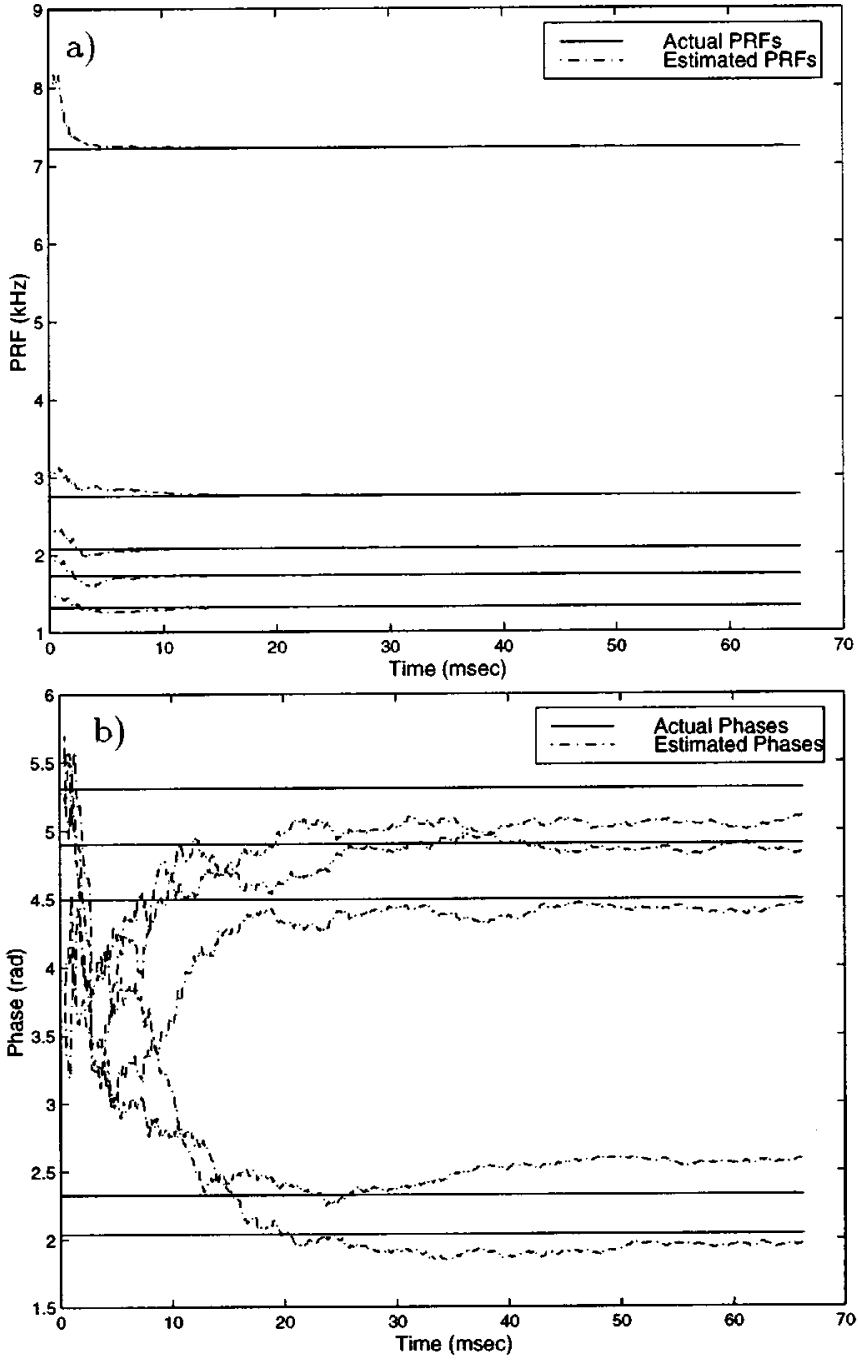

Fig. 7. Parameter estimation over 1000 pulses in the presence of noise with known variance $\sigma^{2}=1 n s^{2}$. (a) Actual and estimated PRFs. (b) Actual and estimated phases.

TABLE IV

COMPARISON OF PRFS IN THE CASE OF ESTIMATION FAILURE

\begin{tabular}{cccc}
\hline Train No. & $\begin{array}{c}\text { Actual PRF } \\
(\mathrm{kHz})\end{array}$ & $\begin{array}{c}\text { Est. PRF } \\
(\mathrm{kHz})\end{array}$ & $\begin{array}{c}\text { Error } \\
(\%)\end{array}$ \\
\hline $\mathbf{1}$ & $\mathbf{1 . 1 2 6 8}$ & $\mathbf{1 . 1 9 9 0}$ & $\mathbf{6 . 4 1}$ \\
$\mathbf{2}$ & $\mathbf{1 . 2 7 1 5}$ & $\mathbf{1 . 1 9 9 2}$ & $\mathbf{5 . 6 9}$ \\
3 & 3.9194 & 3.9199 & $2.6 \mathrm{e}-3$ \\
4 & 5.0299 & 5.0300 & $\mathbf{1 . 4 e - 3}$ \\
\hline
\end{tabular}

TABLE V

Comparison of Phases in the CASE of Estimation Failure

\begin{tabular}{cccc}
\hline Train No. & $\begin{array}{c}\text { Actual Phase } \\
\text { (rad) }\end{array}$ & $\begin{array}{c}\text { Est. Phase } \\
\text { (rad) }\end{array}$ & $\begin{array}{c}\text { Error } \\
(\%)\end{array}$ \\
\hline 1 & $\mathbf{0 . 2 1}$ & $\mathbf{1 . 4 4}$ & $\mathbf{1 9 . 5}$ \\
$\mathbf{2}$ & $\mathbf{5 . 9 2}$ & $\mathbf{4 . 6 4}$ & $\mathbf{2 0 . 3}$ \\
$\mathbf{3}$ & 6.10 & 6.23 & 2.1 \\
$\mathbf{4}$ & 0.61 & 0.73 & 1.9 \\
\hline
\end{tabular}

\section{CONCLUSION}

An important aspect of this scheme for estimating the parameters of interleaved pulse trains is its computational efficiency. If there are $N$ pulses to be processed in the interleaved train, 
then computations are of order $N$. Since computationally efficient (order $N \log N$ ) fast Fourier transform methods give fairly accurate estimates of the pulse train frequencies [8], this new method is used to fine tune the PRF estimates and yield estimates of phase.

Accurate estimates of both phase and PRF could be useful for deinterleaving periodic pulse trains. With the method applied here, the phases of up to seven interleaved trains can be estimated to around $2 \%$ of their actual values if 2000 pulses are processed. The PRF estimates can be fine tuned from a $10 \%$ error to virtually zero (around $15 \mathrm{e}-3 \%$ ) error.

This method is robust to significant jitter noise on the pulse train times of arrival. Naturally, as more noise is added, performance degrades with fewer train parameters that can be estimated and greater errors in the estimation.

Of course, in hindsight, the proposed method is a straightforward application of the familiar extended Kalman filter. The significance of this work is that this tool is able to achieve such useful results in a context in which a priori, one would not expect it to perform well since it approximates discontinuities by smooth functions. Our experience is that the nature of the smoothness approximations is critical, and one approximation at least, as studied here, is seen to be quite facilitating.

\section{REFERENCES}

[1] R. G. Wiley, Electronic Intelligence: The Analysis of Radar Signals. Norwell, MA: Artech House, 1993.

[2] H. K. Mardia, "New techniques for the deinterleaving of repetitive sequences," Proc. Inst. Elect. Eng. F, vol. 136, pp. 149-154, 1989.

[3] D. J. Milojević and B. M. Popović, "Improved algorithm for the deinterleaving of radar pulses," Proc. Inst. Elect. Eng. F, vol. 139, pp. 98-104, 1992.

[4] J. B. Moore and V. Krishnamurthy, "Deinterleaving pulse trains using discrete-time stochastic dynamic-linear models," IEEE Trans Signal Processing, vol. 42, pp. 3092-3103, Nov. 1994.

[5] B. D. O. Anderson and J. B. Moore, Optimal Filtering. Englewood Cliffs, NJ: Prentice-Hall, 1979, ch. 8, pp. 193-205.

[6] T. Conroy and J. B. Moore, "The limits of extended Kalman filtering for pulse train deinterleaving," IEEE Trans Signal Processing, vol. 46, pp. 3326-3332, Dec. 1998

[7] E. T. Kofler and C. T. Leondes, "New approach to the pulse train de-interleaving problem," Int. J. Syst. Sci., vol. 20, no. 12, pp. 2663-2671, 1989.

[8] R. J. Orsi, J. B. Moore, and R. E. Mahony, "Spectrum estimation of interleaved pulse trains," in Proc ISSPA, vol. 2, Gold Coast, Australia, 1996, pp. $125-128$
[9] D. A. Gray, B. J. Slocumb, and S. D. Elton, "Parameter estimation for periodic discrete event processes," in Proc. Int. Conf. Acoust., Speech Signal Process., vol. 4, 1994, pp. 93-96.

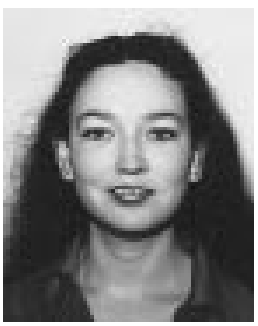

Tanya L. Conroy received the B.Sc. and B.E. degrees in 1993 and 1995, respectively, all from the Australian National University (ANU), Canberra. She recently completed work toward the Ph.D degree in systems engineering at ANU.

Her research interests include signal processing and robotic vision systems.

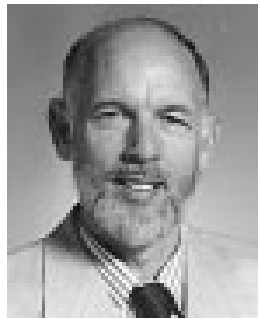

John B. Moore (F'79) was born in China in 1941. He received the B.E., M.E., and Ph.D. degrees in 1963, 1964, and 1967, respectively, all in electrical engineering from the University of Santa Clara, Santa Clara, CA.

He was appointed Senior Lecturer at the Electrical Engineering Department, University of Newcastle, Callaghan, Australia, in 1967, and then promoted to Associate Professor in 1968 and full Professor (personal chair) in 1973. He was Department Head from 1975 to 1979. In 1982, he was appointed Professorial Fellow in the Department of Systems Engineering, Research School of Physical Sciences, Australian National University, Canberra, and was promoted to Professor in 1990. He was Head of the Department from 1992 to 1999. He has also spent periods in industry as a Design Engineer and as a Consultant and has had research grants from industry and government laboratories, including as a named Researcher in the Co-Operative Research Centre for Robust and Adaptive Systems. His current research is in control systems, optimization, and signal processing with application areas to robotic and vision systems, GPS/INS navigation, and communication systems. He is Co-Author, with B. Anderson, of three books: Linear Optimal Control (Englewood Cliffs, NJ: Prentice-Hall, 1971), Optimal Filtering (Englewood Cliffs, NJ: Prentice-Hall, 1979), and Optimal Control-Linear Quadratic Methods (Englewood Cliffs, NJ: Prentice-Hall, 1989). He was Co-Author of the book, with U. Helmke, Optimization and Dynamical Systems (New York: Springer-Verlag, 1993). He co-wrote the book, with L. Aggoun and R. J. Elliott, Hidden Markov Model Estimation and Control via Reference Methods (New York: Springer-Verlag, 1995) and the book, with T. T. Tay and I. Mareels, High Performance Control (Boston MA: Birkhauser 1997).

Prof. Moore is a Fellow of the Australian Academy of Technological Sciences and Engineering and a Fellow of the Australian Academy of Science. 